\title{
Studying the Interrelationship amongst Barriers to Internationalization of SMEs in Developing Countries like India
}

\author{
Arnav Jain \\ Techture Structures Private \\ Limited \\ Indore, India
}

\author{
Remica Aggarwal \\ School of Business, University of \\ Petroleum \& Energy Studies \\ Dehradun, India
}

\author{
Veena Aggarwal \\ Recventures Education Services \\ Private Limited \\ Delhi, India
}

\begin{abstract}
The enterprises that do business exclusively in domestic markets do not have a long-term future in the global market so internationalization is not only the need but also the necessity for most companies. Companies as well as countries can reap numerous benefits from this process but there are also barriers that slow down the process and make it harder. Following research discusses various barriers to internationalization of SME $s$ in developing countries such as India. Further the interrelationship amongst these barriers has been discussed using ISM methodology.
\end{abstract}

\section{Keywords}

ISM methodology; Internationalization of enterprise construction industry; SMEs; business internationalization

\section{INTRODUCTION}

The SME sector is of crucial importance to national economies. Not only in developed countries such as Europe and United States but also in developing countries it enjoy the status of delivering a majority of business activities. The SMEs usually enjoys the advantages of flexibility, openness, dynamism and innovation, but may also at the same time faces various barriers and therefore to keep a balance and to maintain stability, it is required that they should pay attention to ever growing competitiveness that originates from various internal and external factors. Global differences in manufacturing conditions as well as innovation, acceptance of new technologies, knowledge of the market and access to funding are basic categories that the management of any SME should special look after. Business internationalization is becoming an important precondition for further growth and development. The advantages of internationalization for both the enterprise and the country are multiple and that is why the executive government must not act as a passive bystander but rather become an active participant, especially when it comes to creating favorable business conditions. The internationalization of enterprise is an extremely complex process that cannot be accomplished overnight and in order to achieve it, one has to be quite educated on the subject and plan it systematically while also having financial means, audacity and just a bit of luck.

The internationalization of business can be defined as a series of business activities outside national borders which are based on applying the notion of international marketing. It has to be emphasized that the internationalization of business is a process that happens both rapidly and in stages, and leads to major changes in the enterprise as well as in the economic activities. Making a decision to internationalize depends on certain preconditions, such as size of the domestic market, market position and enterprise flexibility; openness of the domestic market; development of the sector; management capability etc. The basic motives for which small and medium sized enterprises internationalize are because of unique product, technological advantage over competition; achieving economies of scale ; to avoid missing on potential business opportunities in foreign markets ; to retain its market share and to strengthen the enterprise's sales outside the national borders. Additional revenue from foreign markets undoubtedly enables normal growth and development of the enterprise. By expanding, it gains access to new technologies and experience in international business, and adopts new skills as well as an addition to the revenue from foreign markets. Researchers can explored further on internationalization of SMEs from [[1]-[16]].

However the barriers are many. The greatest limitations to the internationalization of business that SMEs have to face are lack of entrepreneurial, managerial and marketing skills. The majority of owners-managers and novice entrepreneurs are experts in the products and services that their company deals with. The fact that they often lack broader managing skills has a negative impact on their long-term success. Strategic planning, marketing, finding buyers, innovation managing, quality dedication, foreign language competence, cash-flow management, government bureaucracy, information technology and many other elements are necessary to face the challenges of the international market successfully. This paper explores the barriers to the internationalization of SMEs in developing countries and further studies the interrelationship amongst them through ISM methodology.

The paper is organized as follows. Section 2 deals with literature review. Section 3 explains the ISM methodology. Section 4 applies the methodology to the case example and section 5 presents the managerial implications.

\section{LITERATURE REVIEW}

Following section presents the literature review on the various barriers to internationalization of SMEs as depicted by literature. There are a number of studies which have focused on the barriers to internationalization $([18],[19])$. Further, the perception of the barriers may vary in intensity depending on the level of internationalization of the individual firm [19]. Limitations of financial and physical resources continuously highlighted as a barrier to internationalization of SMEs ([20],[21]). Lack of capital requirements and other firm resources and limited access to key infrastructure were also reported by small and medium-sized enterprises. Small and medium-sized enterprises are unique and different from large enterprises, particularly the availability of resources of the organization to manage the SME is different from the 
management of a larger business. Authors have also made use of Google search scholar, Mendeley software, and other search engines and softwares to explore the literature and get the content. Various keywords such as internationalization of small and medium sized enterprises, small medium enterprises, barriers to internationalization etc. have been used over these search engines. Following table I compile the major categories of barriers as depicted by literature.

\section{Table I: Barriers to internationalization of SME s}

\begin{tabular}{|c|c|c|}
\hline 1. & $\begin{array}{c}\text { Financial } \\
\text { barriers }\end{array}$ & 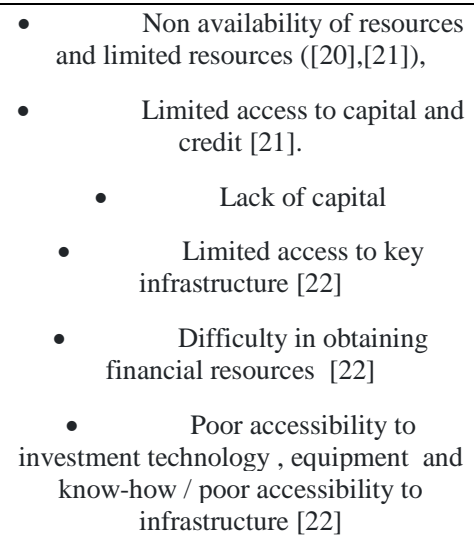 \\
\hline 2. & $\begin{array}{c}\text { Managerial } \\
\text { barriers }\end{array}$ & 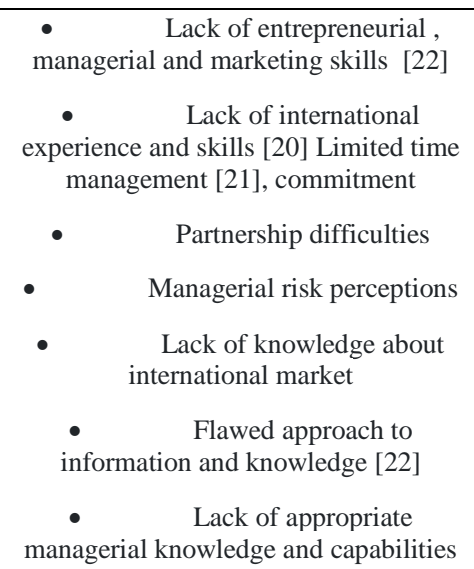 \\
\hline 3. & $\begin{array}{c}\text { Market } \\
\text { based } \\
\text { barriers }\end{array}$ & $\begin{array}{c}\text { Government regulation, } \\
\text { including tariff and non-tariff barriers } \\
\text { (McDougall, 1989; Coviello \& McAuley, } \\
\text { 1999), } \\
\text { Lack of market knowledge } \\
\text { and cultural differences or psychic } \\
\text { distance [20], } \\
\text { Strong domestic market }\end{array}$ \\
\hline 4. & $\begin{array}{l}\text { Industry } \\
\text { specific } \\
\text { barriers }\end{array}$ & $\begin{array}{l}-\quad \begin{array}{l}\text { Competition of indigenous } \\
\text { companies [22] }\end{array} \\
\text { Change in market structure } \\
\text { - } \quad \text { High technology cost }\end{array}$ \\
\hline 5. & $\begin{array}{c}\text { Firm } \\
\text { specific } \\
\text { barriers }\end{array}$ & $\begin{array}{c}\text { Difficulties related to } \\
\text { organizational resources and capabilities } \\
\text { [18] } \\
\text { Difficulty in contacting } \\
\text { foreign customers } \\
\text { inefficiencies in functions of the firm } \\
\text { Functional barriers refer to } \\
\text { Standardization discrepancies } \\
\text { [22] }\end{array}$ \\
\hline
\end{tabular}

\begin{tabular}{|c|c|c|}
\hline & & $\begin{array}{l}\text { Risk of selling abroad } \\
\text { Marketing barriers such as } \\
\text { difficulty in finding the right distribution } \\
\text { channel } \\
\text { Difficulty in locating and } \\
\text { analyzing foreign markets, finding } \\
\text { international market date, identifying } \\
\text { foreign business opportunities, and } \\
\text { contacting foreign customers } \\
\text { ([2],[3],[17]) }\end{array}$ \\
\hline 6. & $\begin{array}{l}\text { Socio- } \\
\text { cultural } \\
\text { barriers }\end{array}$ & $\begin{array}{l}\text { Language barriers and cultural } \\
\text { differences }\end{array}$ \\
\hline 7. & $\begin{array}{l}\text { External } \\
\text { barriers }\end{array}$ & $\begin{array}{l}\text { Procedural barriers such as } \\
\text { operational aspects of transactions with } \\
\text { foreign customers, unfamiliarity with } \\
\text { techniques and procedures } \\
\text { communication failures, and the slow } \\
\text { collection of payments } \\
\text { - Home and host country } \\
\text { environment the firm operates in [18] } \\
\text { - } \quad \text { Foreign country priorities in } \\
\text { the internationalization Process [23-26] } \\
\text { - Governmental barriers such as } \\
\text { bureaucracy lack of government } \\
\text { incentives }\end{array}$ \\
\hline
\end{tabular}

Based on the literature review, following barriers have been selected and studied further to find the inter-relationship amongst them based on ISM methodology.

1. Lack of entrepreneurial, managerial and marketing skills (LEMM)

2. Lack of government incentives and bureaucracy (LGIB)

3. Legal barriers which include inadequate intellectual property protection etc. (LB)

4. Socio - cultural barriers (SCB) such as language barriers and cultural differences

5. Competition amongst companies (CAC)

6. Lack of financial capital (LFC)

7. Inadequate financial and human resources which includes limited access to financial and human assistance (IFHR)

8. Poor accessibility to infrastructure and equipments (PAI)

9. Lack of knowledge and information (LKI)

10. Standardization discrepancies and quality management issues (SDQM)

11. Complicated trade documentation, labeling and packaging (CTD)

12. Market based barriers (MBB) such as risk of selling abroad and cost of overseas operations

13. High technology costs /poor accessibility to investment in technology (HTC)

14. Unfamiliarity with techniques and procedures (UTP) 


\section{INTERPRETIVE STRUCTURAL}

\section{MODELLING METHODOLOGY}

Interpretive Structural Modeling or ISM first proposed by [27] is a computer assisted learning process that enables the researcher to develop a map of the complex relationships between the many elements involved in a complex situation. In this technique a set of unique interrelated variables are structured in the form of a hierarchy graph called the diagraph. Its steps are as follows: Firstly, identify the relevant elements and establish a contextual relationship amongst them. Thereafter, develop a structural self-interaction matrix (SSIM) using $\mathrm{V}, \mathrm{A}, \mathrm{X} \& \mathrm{O}$ where the symbols have the following meanings i.e. $\mathrm{V}$ for the relation from $\mathrm{i}$ to $\mathrm{j}$ but not in both directions; A for the relation from $\mathrm{j}$ to $\mathrm{i}$ but not in both directions; $\mathrm{X}$ for both direction relations from $\mathrm{i}$ to $\mathrm{j}$ and $\mathrm{j}$ to $\mathrm{i}$; and $\mathrm{O}$ if the relation between the variables does not appear valid. Using SSIM, initial reachability matrix can be formed which has all values in binary form. A final reachability matrix is formed after removing transitivity from initial reachability matrix. From the final reachability matrix, the reachability set and antecedent set for each criterion and for each element is found [27]. The element for which the reachability and intersection sets are the same is the top-level element. At every iteration a top level element is identified which is removed in the next iteration. After all the elements have been identified at different level of hierarchy, a Mic-Mac analysis (based on the driving power and dependence power) and a diagraph can be formed.

\section{DEVELOPMENT OF ISM MODEL}

In this section, ISM model is developed for studying the interrelationships amongst various barriers to internationalization of SMEs in developing countries. Fourteen important criteria are Lack of entrepreneurial, managerial and marketing skills (LEMM); Lack of government incentives and bureaucracy (LGIB); Legal and political barriers (LPB); Socio - cultural barriers (SCB) ; Competition amongst companies (CAC) ; Lack of financial capital (LFC) ; Inadequate financial and human resources (lack of intelligent and qualified staff) (IFHR) ; Poor accessibility to infrastructure and equipments (PAI) ; Lack of knowledge and information (LKI) ; Standardization discrepancies and quality management issues (SDQM); Complicated trade documentation (CTD); Market based barriers (MBB) ; High technology costs (HTC) ; Unfamilarity with techniques and procedures (UTP).

\subsection{Construction of Structural Self - Interaction Matrix (SSIM)}

This matrix gives the pair-wise relationship between two variables i.e. $\mathrm{i}$ and $\mathrm{j}$ based on VAXO. SSIM has been presented below in Fig 1.

\subsection{Construction of Initial reachability matrix and final reachability matrix}

The SSIM has been converted in to a binary matrix called the initial reachability matrix shown in fig. 2 by substituting $\mathrm{V}, \mathrm{A}$, $\mathrm{X}, \mathrm{O}$ by 1 or 0 as per the case. After incorporating the transitivity, the final reachability matrix is shown below in the Fig 3.

Fig 1: SSIM matrix for pair wise relationship amongst barriers

\begin{tabular}{|c|c|c|c|c|c|c|c|c|c|c|c|c|c|c|c|}
\hline Barrier & & 1 & 2 & 3 & 4 & 5 & 6 & 7 & 8 & 9 & 10 & 11 & 12 & 13 & 14 \\
\hline & & $\begin{array}{c}\mathrm{LE} \\
\mathrm{M} \\
\mathrm{M}\end{array}$ & $\begin{array}{c}\text { LGI } \\
\text { B }\end{array}$ & $\begin{array}{c}\text { LP } \\
\text { B }\end{array}$ & SCB & CAC & $\begin{array}{c}\text { LF } \\
\text { C }\end{array}$ & $\begin{array}{c}\text { IFH } \\
\text { R }\end{array}$ & $\begin{array}{c}\text { PA } \\
\text { I }\end{array}$ & $\begin{array}{c}\text { LK } \\
\text { I }\end{array}$ & $\begin{array}{c}\text { SDQ } \\
M\end{array}$ & $\begin{array}{c}\text { CT } \\
\text { D }\end{array}$ & $\begin{array}{c}\mathrm{MB} \\
\mathrm{B}\end{array}$ & $\begin{array}{c}\text { HT } \\
\text { C }\end{array}$ & UTP \\
\hline 1. & LEMM & & $\mathrm{A}$ & $\mathrm{V}$ & V & $\mathrm{O}$ & A & $\mathrm{A}$ & A & $\mathrm{V}$ & V & $\mathrm{V}$ & $\mathrm{V}$ & $\mathrm{V}$ & $\mathrm{X}$ \\
\hline 2. & LGIB & & & $\mathrm{O}$ & $\mathrm{V}$ & $\mathrm{V}$ & $\mathrm{V}$ & $\mathrm{V}$ & $\mathrm{V}$ & $\mathrm{V}$ & $\mathrm{V}$ & $\mathrm{V}$ & $\mathrm{V}$ & $\mathrm{V}$ & $\mathrm{V}$ \\
\hline 3. & LPB & & & & $\mathrm{O}$ & V & $\mathrm{O}$ & A & A & $\mathrm{A}$ & V & V & $X$ & V & $\mathrm{A}$ \\
\hline 4. & SCB & & & & & $\mathrm{V}$ & A & A & A & A & $\mathrm{V}$ & V & $\mathrm{V}$ & $\mathrm{V}$ & A \\
\hline 5. & CAC & & & & & & $\mathrm{O}$ & $\mathrm{A}$ & $\mathrm{O}$ & $\mathrm{O}$ & $\mathrm{A}$ & $\mathrm{A}$ & $\mathrm{A}$ & $\mathrm{A}$ & $\mathrm{O}$ \\
\hline 6. & LFC & & & & & & & $\mathrm{V}$ & $\mathrm{V}$ & $\mathrm{V}$ & V & $\mathrm{V}$ & A & $\mathrm{V}$ & $\mathrm{O}$ \\
\hline 7. & IFHR & & & & & & & & $\mathrm{V}$ & $\mathrm{V}$ & $\mathrm{V}$ & $\mathrm{V}$ & $\mathrm{V}$ & $\mathrm{V}$ & $\mathrm{V}$ \\
\hline 8. & PAI & & & & & & & & & $X$ & $\mathrm{~V}$ & $\mathrm{~V}$ & $\mathrm{~V}$ & $\mathrm{~V}$ & $\mathrm{X}$ \\
\hline 9. & LKI & & & & & & & & & & $\mathrm{V}$ & $\mathrm{V}$ & $\mathrm{V}$ & $\mathrm{V}$ & $\mathrm{X}$ \\
\hline 10. & SDQM & & & & & & & & & & & $\mathrm{X}$ & A & $\mathrm{V}$ & A \\
\hline 11. & CTD & & & & & & & & & & & & $\mathrm{A}$ & $\mathrm{V}$ & $\bar{A}$ \\
\hline 12. & MBB & & & & & & & & & & & & & $\mathrm{V}$ & A \\
\hline 13. & HTC & & & & & & & & & & & & & & A \\
\hline 14. & UTP & & & & & & & & & & & & & & \\
\hline
\end{tabular}


Fig 2: Initial reachability matrix

\begin{tabular}{|c|c|c|c|c|c|c|c|c|c|c|c|c|c|c|c|}
\hline Barri & & 1 & 2 & 3 & 4 & 5 & 6 & 7 & 8 & 9 & 10 & 11 & 12 & 13 & 14 \\
\hline & & $\begin{array}{c}\text { LEM } \\
\text { M }\end{array}$ & $\begin{array}{c}\text { LGI } \\
\text { B }\end{array}$ & $\begin{array}{c}\text { LP } \\
\text { B }\end{array}$ & $\mathrm{SCB}$ & $\mathrm{CAC}$ & LFC & IFHR & PAI & $\begin{array}{c}\text { LK } \\
\text { I }\end{array}$ & $\begin{array}{c}\text { SDQ } \\
\text { M }\end{array}$ & $\begin{array}{c}\text { CT } \\
\text { D }\end{array}$ & $\begin{array}{c}\mathrm{MB} \\
\mathrm{B}\end{array}$ & $\begin{array}{c}\text { HT } \\
\text { C }\end{array}$ & UTP \\
\hline 1. & LEMM & 1 & 0 & 1 & 1 & 0 & 0 & 0 & 0 & 1 & 1 & 1 & 1 & 1 & 1 \\
\hline 2. & LGIB & 1 & 1 & 0 & 1 & 1 & 1 & 1 & 1 & 1 & 1 & 1 & 1 & 1 & 1 \\
\hline 3. & LPB & 0 & 0 & 1 & 0 & 1 & 0 & 0 & 0 & 0 & 1 & 1 & 1 & 1 & 0 \\
\hline 4. & SCB & 0 & 0 & 0 & 1 & 1 & 0 & 0 & 0 & 0 & 1 & 1 & 1 & 1 & 0 \\
\hline 5. & $\mathrm{CAC}$ & 0 & 0 & 0 & 0 & 1 & 0 & 0 & 0 & 0 & 0 & 0 & 0 & 0 & 0 \\
\hline 6. & LFC & 1 & 0 & 0 & 1 & 0 & 1 & 1 & 1 & 1 & 1 & 1 & 0 & 1 & 0 \\
\hline 7. & IFHR & 1 & 0 & 1 & 1 & 1 & 0 & 1 & 1 & 1 & 1 & 1 & 1 & 1 & 1 \\
\hline 8. & PAI & 1 & 0 & 1 & 1 & 0 & 0 & 1 & 1 & 1 & 1 & 1 & 1 & 1 & 1 \\
\hline 9. & LKI & 0 & 0 & 0 & 1 & 0 & 0 & 0 & 1 & 1 & 1 & 1 & 1 & 1 & 1 \\
\hline 10. & SDQM & 0 & 0 & 0 & 0 & 1 & 0 & 0 & 0 & 0 & 1 & 1 & 0 & 1 & 0 \\
\hline 11. & CTD & 0 & 0 & 1 & 0 & 1 & 0 & 0 & 0 & 0 & 1 & 1 & 0 & 1 & 0 \\
\hline 12. & MBB & 0 & 0 & 0 & 0 & 1 & 0 & 0 & 0 & 0 & 1 & 1 & 1 & 1 & 0 \\
\hline 13. & HTC & 0 & 0 & 1 & 0 & 1 & 1 & 0 & 0 & 0 & 0 & 0 & 0 & 1 & 0 \\
\hline 14. & UTP & 1 & 0 & 1 & 1 & 0 & 0 & 0 & 1 & 1 & 1 & 1 & 1 & 1 & 1 \\
\hline
\end{tabular}

Fig 3 : Final reachability matrix

\begin{tabular}{|c|c|c|c|c|c|c|c|c|c|c|c|c|c|c|c|c|}
\hline Barri & & 1 & 2 & 3 & 4 & 5 & 6 & 7 & 8 & 9 & 10 & 11 & 12 & 13 & 14 & \\
\hline & & $\begin{array}{c}\text { LEM } \\
\text { M }\end{array}$ & $\begin{array}{c}\text { LGI } \\
\text { B }\end{array}$ & $\begin{array}{c}\text { LP } \\
\text { B }\end{array}$ & $\mathrm{SCB}$ & CAC & LFC & IFHR & PAI & $\begin{array}{c}\text { LK } \\
\text { I }\end{array}$ & $\begin{array}{c}\text { SDQ } \\
\mathrm{M}\end{array}$ & $\begin{array}{c}\mathrm{CT} \\
\mathrm{D}\end{array}$ & $\begin{array}{c}\text { MB } \\
\text { B }\end{array}$ & $\begin{array}{c}\mathrm{HT} \\
\mathrm{C}\end{array}$ & UTP & D.P \\
\hline 1. & LEMM & 1 & 0 & 1 & 1 & 1 & 0 & 0 & 1 & 1 & 1 & 1 & 1 & 1 & 1 & 11 \\
\hline 2. & LGIB & 1 & 1 & 1 & 1 & 1 & 1 & 1 & 1 & 1 & 1 & 1 & 1 & 1 & 1 & 14 \\
\hline 3. & LPB & 0 & 0 & 1 & 0 & 1 & 0 & 0 & 0 & 0 & 1 & 1 & 1 & 1 & 0 & 6 \\
\hline 4. & SCB & 0 & 0 & 1 & 1 & 1 & 1 & 0 & 0 & 0 & 1 & 1 & 1 & 1 & 0 & 8 \\
\hline 5. & $\mathrm{CAC}$ & 0 & 0 & 0 & 0 & 1 & 0 & 0 & 0 & 0 & 0 & 0 & 0 & 0 & 0 & 1 \\
\hline 6. & LFC & 1 & 0 & 0 & 1 & 0 & 1 & 1 & 1 & 1 & 1 & 1 & 0 & 1 & 1 & 10 \\
\hline 7. & IFHR & 1 & 0 & 1 & 1 & 1 & 0 & 1 & 1 & 1 & 1 & 1 & 1 & 1 & 1 & 12 \\
\hline 8. & PAI & 1 & 0 & 1 & 1 & 1 & 0 & 1 & 1 & 1 & 1 & 1 & 1 & 1 & 1 & 12 \\
\hline 9. & LKI & 0 & 0 & 0 & 1 & 1 & 0 & 0 & 1 & 1 & 1 & 1 & 1 & 1 & 1 & 9 \\
\hline 10. & SDQM & 0 & 0 & 1 & 0 & 1 & 1 & 0 & 0 & 0 & 1 & 1 & 0 & 1 & 0 & 6 \\
\hline 11. & CTD & 0 & 0 & 1 & 0 & 1 & 1 & 0 & 0 & 0 & 1 & 1 & 1 & 1 & 0 & 7 \\
\hline 12. & MBB & 0 & 0 & 0 & 0 & 1 & 0 & 0 & 0 & 0 & 1 & 1 & 1 & 1 & 0 & 5 \\
\hline 13. & HTC & 0 & 0 & 1 & 0 & 1 & 1 & 0 & 0 & 0 & 1 & 0 & 0 & 1 & 0 & 5 \\
\hline \multirow[t]{2}{*}{14.} & UTP & 1 & 0 & 1 & 1 & 1 & 0 & 0 & 1 & 1 & 1 & 1 & 1 & 1 & 1 & 11 \\
\hline & De.P & 6 & 1 & 10 & 8 & 13 & 6 & 4 & 7 & 7 & 13 & 12 & 10 & 13 & 7 & \\
\hline
\end{tabular}




\subsection{Level Partition}

Table 3 : Iteration I

\begin{tabular}{|c|c|c|c|c|}
\hline $\begin{array}{l}\text { S. } \\
\text { No }\end{array}$ & $\begin{array}{c}\text { Reachability } \\
\text { set }\end{array}$ & $\begin{array}{c}\text { Antecedent } \\
\text { set }\end{array}$ & $\begin{array}{l}\text { Intersectio } \\
\quad \text { n set }\end{array}$ & $\begin{array}{c}\text { Iteratio } \\
\mathbf{n}\end{array}$ \\
\hline 1 & $5,10,13$ & $\begin{array}{c}1,2,3,4,7,8,9 \\
10,11,12,13\end{array}$ & 10,13 & \\
\hline 2 & 5,11 & $\begin{array}{c}1,2,3,4,7,8,9 \\
10,11,12,14\end{array}$ & 11 & \\
\hline 3 & 5 & $\begin{array}{c}1,2,3,4,5,7,8 \\
9,10,11,12,13 \\
, 14\end{array}$ & 5 & \\
\hline 4 & $3,10,11,13$ & $\begin{array}{c}1,2,3,4,7,8,10 \\
, 11,13,14\end{array}$ & $3,10,11,13$ & \\
\hline 5 & $10,11,12,13$ & $\begin{array}{c}1,2,3,4,6,7,8 \\
9,11,12,14\end{array}$ & 11,12 & I \\
\hline 6 & $\begin{array}{c}3,4,10,11,12,1 \\
3\end{array}$ & $1,2,3,4,7,8$ & 3,4 & \\
\hline 7 & $\begin{array}{c}1,4,6,8,9,10,1 \\
1,12,13,14\end{array}$ & $1,2,7,8,9,14$ & $8,9,14$ & \\
\hline 8 & 1,14 & $1,2,7,8,14$ & 1,14 & \\
\hline 9 & $1,6,7$ & 2,6 & 6 & \\
\hline 10 & 1,7 & $2,6,7,8$ & 7 & \\
\hline 11 & $\begin{array}{c}1,2,3,4,5,6,7,8 \\
, 9,10,11,12,13 \\
, 14\end{array}$ & 2 & 2 & \\
\hline 12 & $1,6,7,8,9,14$ & 2,6 & 6 & \\
\hline
\end{tabular}

From the final reachability matrix, reachability and final antecedent set for each factor are found. The element for which the reachability and intersection sets are same are the top-level element in the ISM hierarchy. After the identification of top level element, it is separated out from the other elements and the process continues for next level of elements. Reachability set, antecedent set, intersection set along with different level for elements have been shown below in table $\mathrm{V}$ to table XI.

Table 4 : Iteration II

\begin{tabular}{|c|c|c|c|c|}
\hline S.No. & $\begin{array}{l}\text { Reachabili } \\
\text { ty set }\end{array}$ & Antecedent set & $\begin{array}{c}\text { Intersection } \\
\text { set }\end{array}$ & $\begin{array}{l}\text { Itera } \\
\text { tion }\end{array}$ \\
\hline 1 & 10,13 & $\begin{array}{c}1,2,3,4,7,8,9,10 \\
, 11,12,13\end{array}$ & 10,13 & \\
\hline 2 & 11 & $\begin{array}{c}1,2,3,4,7,8,9,10 \\
, 11,12,14\end{array}$ & 11 & \\
\hline 4 & $3,10,11,13$ & $\begin{array}{c}1,2,3,4,7,8,10,1 \\
1,13,14\end{array}$ & $3,10,11,13$ & \\
\hline 5 & $10,11,12,13$ & $\begin{array}{c}1,2,3,4,6,7,8,9 \\
11,12,14\end{array}$ & 11,12 & \\
\hline 6 & $\begin{array}{c}3,4,10,11,1 \\
2,13\end{array}$ & $1,2,3,4,7,8$ & 3,4 & \\
\hline 7 & $\begin{array}{l}1,4,6,8,9,10 \\
, 11,12,13,1\end{array}$ & $1,2,7,8,9,14$ & $8,9,14$ & II \\
\hline
\end{tabular}

\begin{tabular}{|c|c|c|c|}
\hline & 4 & & \\
\hline 8 & 1,14 & $1,2,7,8,14$ & 1,14 \\
\hline 9 & $1,6,7$ & 2,6 & 6 \\
\hline 10 & 1,7 & $2,6,7,8$ & 7 \\
\hline 11 & $\begin{array}{c}1,2,3,4,6,7, \\
8,9,10,11,1 \\
2,13,14\end{array}$ & 2 & 2 \\
\hline
\end{tabular}

Table 5 : Iteration III

\begin{tabular}{|c|c|c|c|c|}
\hline $\begin{array}{l}\text { Sr. } \\
\text { No. }\end{array}$ & $\begin{array}{c}\text { Reachability } \\
\text { set }\end{array}$ & $\begin{array}{c}\text { Antecedent } \\
\text { set }\end{array}$ & $\begin{array}{c}\text { Intersection } \\
\text { set }\end{array}$ & $\begin{array}{l}\text { Itera } \\
\text { tion }\end{array}$ \\
\hline 4 & 3 & $\begin{array}{c}1,2,3,4,7,8,1 \\
4\end{array}$ & 3 & \multirow{9}{*}{ III } \\
\hline 5 & 12 & $\begin{array}{c}1,2,3,4,6,7,8 \\
9,11,12,14\end{array}$ & 12 & \\
\hline 6 & $3,4,12$ & $1,2,3,4,7,8$ & 3,4 & \\
\hline 7 & $1,4,6,8,9,12,14$ & $1,2,7,8,9,14$ & $8,9,14$ & \\
\hline 8 & 1,14 & $1,2,7,8,14$ & 1,14 & \\
\hline 9 & $1,6,7$ & 2,6 & 6 & \\
\hline 10 & 1,7 & $2,6,7,8$ & 7 & \\
\hline 11 & $\begin{array}{c}1,2,3,4,6,7,8,9, \\
12,14\end{array}$ & 2 & 2 & \\
\hline 12 & $1,6,7,8,9,14$ & 2,6 & 6 & \\
\hline
\end{tabular}

Table 6 : Iteration IV

\begin{tabular}{|c|c|c|c|c|}
\hline $\begin{array}{c}\text { S.N } \\
\text { o. }\end{array}$ & $\begin{array}{l}\text { Reachabilit } \\
\text { y set }\end{array}$ & Antecedent set & $\begin{array}{c}\text { Intersecti } \\
\text { on set }\end{array}$ & $\begin{array}{c}\text { Iterati } \\
\text { on/ } \\
\text { Levels }\end{array}$ \\
\hline 4 & 3 & $1,2,3,4,7,8,14$ & 3 & \multirow{8}{*}{ IV } \\
\hline 6 & 3,4 & $1,2,3,4,7,8$ & 3,4 & \\
\hline 7 & $1,4,6,8,9,14$ & $1,2,7,8,9,14$ & $8,9,14$ & \\
\hline 8 & 1,14 & $1,2,7,8,14$ & 1,14 & \\
\hline 9 & $1,6,7$ & 2,6 & 6 & \\
\hline 10 & 1,7 & $2,6,7,8$ & 7 & \\
\hline 11 & $\begin{array}{c}1,2,3,4,6,7,8 \\
, 9,14\end{array}$ & 2 & 2 & \\
\hline 12 & $1,6,7,8,9,14$ & 2,6 & 6 & \\
\hline
\end{tabular}


Table 7 : Iteration V

\begin{tabular}{|c|c|c|c|c|}
\hline $\begin{array}{c}\text { Sr. } \\
\text { No. }\end{array}$ & $\begin{array}{c}\text { Reachability } \\
\text { set }\end{array}$ & $\begin{array}{c}\text { Antecedent } \\
\text { set }\end{array}$ & $\begin{array}{c}\text { Intersection } \\
\text { set }\end{array}$ & $\begin{array}{c}\text { Itera } \\
\text { tion }\end{array}$ \\
\hline 6 & $\mathbf{4}$ & $1,2,4,7,8$ & $\mathbf{4}$ & \\
\hline 7 & $1,4,8,9,14$ & $1,2,7,8,14$ & $1,8,9,14$ & \\
\hline 8 & 1,14 & $1,2,7,8,14$ & 1,14 & \multirow{2}{*}{$\mathbf{V}$} \\
\hline 9 & $1,6,7$ & 2,6 & 6 & \\
\hline 10 & 1,7 & $2,6,7,8$ & 7 & \\
\hline 11 & $1,2,4,6,7,8,9,1$ & 2 & 2 & \\
\hline 12 & 4 & & & \\
\hline
\end{tabular}

Table 8 : Iteration VI

\begin{tabular}{|c|c|c|c|c|}
\hline $\begin{array}{l}\text { Sr. } \\
\text { No. }\end{array}$ & $\begin{array}{c}\text { Reachability } \\
\text { set }\end{array}$ & Antecedent set & $\begin{array}{c}\text { Intersection } \\
\text { set }\end{array}$ & $\begin{array}{c}\text { Itera } \\
\text { tion }\end{array}$ \\
\hline 7 & $1,8,9,14$ & $1,2,7,8,14$ & $1,8,9,14$ & \multirow{6}{*}{ VI } \\
\hline 8 & 1,14 & $1,2,7,8,14$ & 1,14 & \\
\hline 9 & $1,6,7$ & 2,6 & 6 & \\
\hline 10 & 1,7 & $2,6,7,8$ & 7 & \\
\hline 11 & $1,2,6,7,8,9,14$ & 2 & 2 & \\
\hline 12 & $1,6,7,8,9,14$ & 2,6 & 6 & \\
\hline
\end{tabular}

Table 9 : Iteration VII

\begin{tabular}{|c|c|c|c|c|}
\hline $\begin{array}{c}\text { Sr. } \\
\text { No. }\end{array}$ & $\begin{array}{c}\text { Reachability } \\
\text { set }\end{array}$ & $\begin{array}{c}\text { Antecedent } \\
\text { set }\end{array}$ & $\begin{array}{c}\text { Intersection } \\
\text { set }\end{array}$ & $\begin{array}{c}\text { Itera } \\
\text { tion }\end{array}$ \\
\hline 9 & 6,7 & 2,6 & 6 & \\
& \multirow{2}{*}{ VII } \\
\hline 10 & 7 & $2,6,7,8$ & 7 & \\
\hline 12 & $2,6,7$ & 2 & 2 & \\
\hline
\end{tabular}

Table 10 : Iteration VIII

\begin{tabular}{|c|c|c|c|c|}
\hline $\begin{array}{c}\text { Sr. } \\
\text { No. }\end{array}$ & $\begin{array}{c}\text { Reachability } \\
\text { set }\end{array}$ & $\begin{array}{c}\text { Antecedent } \\
\text { set }\end{array}$ & $\begin{array}{c}\text { Intersection } \\
\text { set }\end{array}$ & $\begin{array}{c}\text { Itera } \\
\text { tion }\end{array}$ \\
\hline 9 & 6 & 2,6 & 6 & \\
\hline 11 & 2,6 & 2 & 2 & \multirow{2}{*}{ VIII } \\
\hline 12 & $\mathbf{6}$ & 2,6 & 6 & \\
\hline
\end{tabular}

Table 11 : Iteration IX

\begin{tabular}{|c|c|c|c|c|}
\hline $\begin{array}{c}\text { Sr. } \\
\text { No. }\end{array}$ & $\begin{array}{c}\text { Reachability } \\
\text { set }\end{array}$ & $\begin{array}{c}\text { Antecedent } \\
\text { set }\end{array}$ & $\begin{array}{c}\text { Intersection } \\
\text { set }\end{array}$ & $\begin{array}{c}\text { Itera } \\
\text { tion }\end{array}$ \\
\hline 11 & $\mathbf{2}$ & 2 & 2 & IX \\
\hline
\end{tabular}

\subsection{Classification of factors}

The critical success factors described earlier are classified in to four clusters viz. autonomous factor, dependent factors, linkage factors and independent factors (mentioned in Table XIII below). As it can be seen that TIDY, SFT and LEC are autonomous criteria. Criteria TEMP, RFM, EC , IC , AR and $\mathrm{MH}$ are drivers . Criteria such as PPC, PR, PB and PrCT are dependent criteria.

Fig. 4 below shows the driving power and dominance diagram.

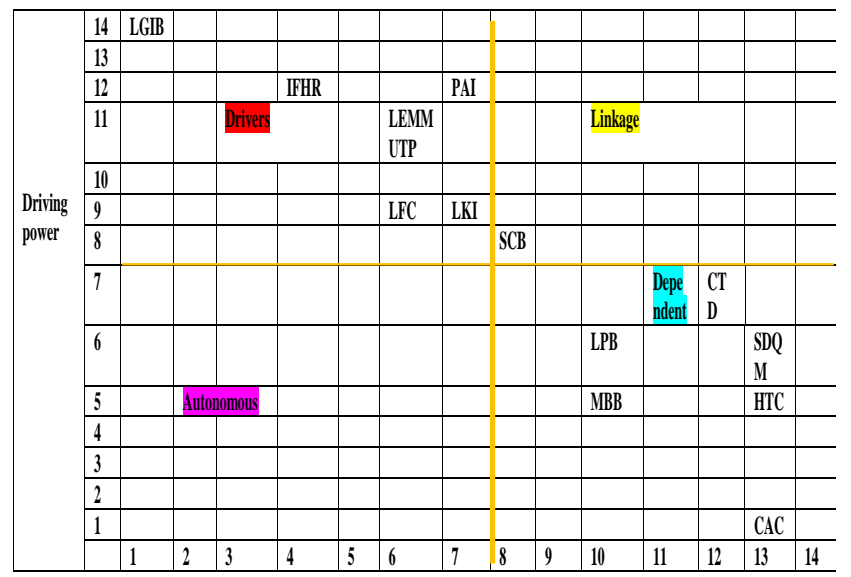

Fig . 4: Driving power and dependence diagram

\subsection{ISM model}

An ISM model is developed ( as shown in fig. 5 below ) after arranging the elements as per their interaction or dependence relationships.

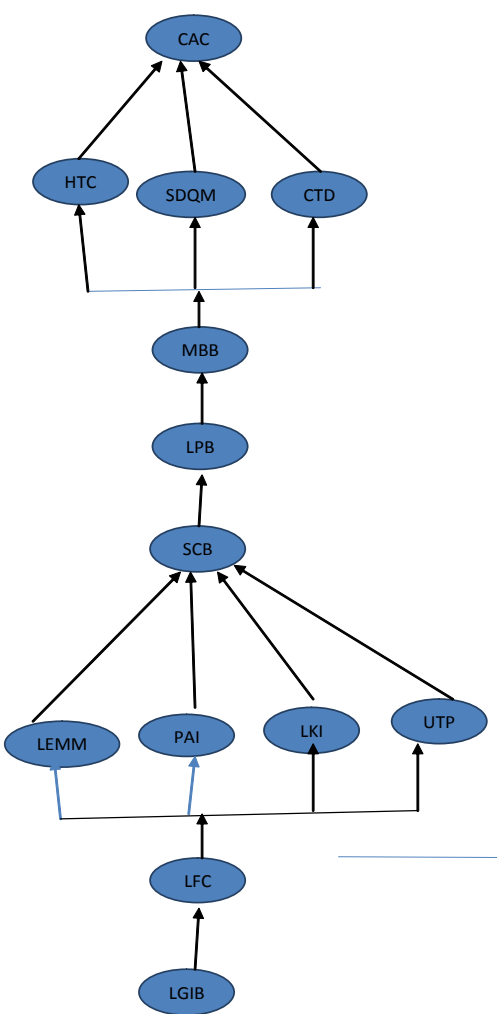

Fig 5: ISM diagraph 


\section{MANAGERIAL IMPLICATIONS AND CONCLUSIONS}

\subsection{Managerial implications}

- Internationalization can be an expensive, timeconsuming and exhausting process for small and medium- sized enterprises, especially if we bear in mind that such enterprises have very limited funds. Therefore, it is very important that the managers take right decisions about the timing and the manner in which to enter a foreign market.

- Educational institutions as well as government organizations and NGOs are also responsible to a great extent towards the internationalization of an organization.

- The enterprise's key motivation as to where, how and why to expand its business to a foreign market lies in the choice and identification of the opportunities by the decision maker and in his/her inclination towards risk. His/her experience and network of contacts plays a crucial role in the development of relevant knowledge.

- Managers must simply understand motives, importance and necessity of being involved in this challenging process. Success in a global environment can only be achieve through dealing with the burden of constant change, identifying key business factors, taking risks but valid and risks in demand, taking risky decisions as per requirement , having a vision and choosing an optimal strategy to make the vision come true and further motivating to implement the set objectives.

\section{ACKNOWLEDGEMENT}

Authors are thankful to Prof. S.P Singh, Department of Management Studies for imparting knowledge on ISM methodology which helped authors substantially while writing this piece of work.

\section{REFERENCES}

[1] Zahra, S., Ireland, R.D. \& Hitt, M. 2000. International expansion by new venture firms: international diversity, mode of market entry, technological learning, and performance, Academy of Management Journal, 43(5), 925-950.

[2] Ruigrok, W. \& Wagner, H. 2003. Internationalization and performance: An organizational learning perspective. Management International Review, 43, 63-83.

[3] Sambharya, R. B. 1995. The combined effect of international and product diversification strategies on the performance of US based multinat on the performance of US based multinational corporations. Management International Review, 35(3), 197-218.

[4] Pangarkar, N. 2008. Internationalization and performance of small- and medium-sized enterprises , Journal of World Business , 43, 475-485.

[5] Pietrasieński P. \& Ślusarczyk B. 2015. Enterprises Empirical Research Review On Barriers To Entry Into Foreign Markets, Polish Journal of Management Studies, 11(1).

[6] Korsakienè R. \& Tvaronavičienè M. 2012. The internationalization of SMEs: an integrative approach,
Journal of Business Economics and Management, 13(2).

[7] Pangarkar, N. \& Lim, H. 2003. Performance of foreign direct investment from Singapore. International Business Review, 12(5), 601-624.

[8] Kennelly, J. J. \& Lewis, E. F. 2002. Degree of internationalization and corporate environmental performance: Is there a link? International Journal of Management, 19(3), 478.

[9] Sullivan, D. 1994. Measuring the degree of internationalization of a firm. Journal of International Business studies, 2Q, 325-342.

[10] Sundaram, A. K. \& Black, J. S. 1992. The environment and internal organization of multinational enterprises. The Academy of Management Review, 17(4), 729-757.

[11] Yip, G. S., Biscarri, J. G. \& Monti, J. A. 2000. The role of the internationalization process in the performance of newly inter of newly internationalizing firms. Journal of International Marketing, 8(3), 10-35.

[12] Stray, S., Bridgewater, S. \& Murray, G. 2001. The internationalization process of small technology based firms: Market choice, mode and degree. Journal of Global Marketing, 15(1), 7.

[13] Riahi-Belkaoui, A. 1998. The effects of the degree of internationalization on firm performance. International Business Review, 7, 315-321.

[14] Rugman, A. M. 1979. International diversification and the multinational enterprise. Lexington, MA: DC Heath.

[15] Rugman, A. M., Lecraw, D. J. \& Booth, L. D. 1985. International business: Firm and the environment. New York: McGraw Hill.

[16] Li, L., Li, D., Dalgic, T. 2004. Internationalization Process of Small and Medium-sized Enterprises: Toward a Hybrid Model of Experiential Learning and Planning, Management International Review, 44(1), 93-116.

[17] Dunning, J. H. 1998. Location and the multinational enterprise: A neglected factor? Journal of International Business Studies, 29(1), 45-66.

[18] Leonidou, L.C. 2004. An Analysis of the Barriers Hindering Small Business Export Development, Journal of Small Business Management, 3.

[19] Morgan, R. E. \& Katsikeas, C. S. 1997. Theories of international trade, foreign direct investment and firm internationalization: A critique. Management Decision, 35(1/2), 68-78.

[20] Karagozoglu, N., \& Lindell, M. 1998. Internationalization of small and medium sized technology based firms: An exploratory study. Journal of Small Business Management, 36(1), 44-59.

[21] Buckley, P. J. 1999. Foreign direct investment by small and medium sized enterprises: The theoretical background. In P. J. Buckley \& P. N. Ghauri(Eds.), The internationalization of the firm, NY: International Thomson Business Press.

[22] Szabo, A. 2002. Internationalization of SMEs, UNECE Entrepreneurship and SME development.

[23] Clark, T. \& Pugh, D. S. 2001. Foreign country priorities in the internationalization process: A measure and an 
exploratory test on British firms. International Business review, 10(3), 285-303.

[24] Calof, J., Beamish, P. 1995. Adapting to foreign markets: explaining internationalisation, International Business Review, 4(2), 115-131.

[25] Collinson, S., Houlden, J. 2005. Decision-Making and Market Orientation in the Internationalization Process of SME, Management International Review, 45, 419-436.
[26] Johanson, J., Vahlne, J.E. 1977. The Internationalization Process of the Firm: A Model of Knowledge Development and Increasing Foreign Market Commitments, Journal of International Business Studies, 8, 23-32.

[27] Warfield, J.N. 1974. Developing interconnection matrices in structural modeling. IEEE Transactions on Systems, Man, and Cybernetics, (1), 81-87. 